\title{
In Silico Analysis and Molecular Modelling of NS2B-NS3 Protein in Dengue
}

\author{
Vinod P. Sinoorkar, Snehal M. Mathe*, Neha Guttikonda \\ Walchand Center for Biotechnology Solapur, Solapur University, Solapur, Maharashtra, India
}

\begin{abstract}
Dengue fever is frequently born viral infection caused by female mosquito Aedes egypti. Transmission of dengue infection is transfer from one host to another by infected mosquito bite. Dengue virus is categorized into various serotypes on the basis of their genetic material variable. Dengue fever and dengue haemorrhagic fever is serious risk factor to Mankind Nowdays there is no specific vaccine to treat the dengue infection. There is need to design potent antiviral vaccine against dengue fever. NS2B-NS3 act as potent drug target in dengue fever. Hence our present work to achieve to understand detail molecular properties of NS2B-NS3 protein by retrieving its amino acid sequence from protein database like PDB. Analysis of physicochemical parameters, secondary and tertiary structure prediction. structure visualization is done by using Rasmol structure visualization tool. Predicted model is validated using procheck analysis. This all information gives primary information for future work to perform computer aided drug design against dengue fever.
\end{abstract}

Keywords : Dengue, Serotypes, NS2B-NS3 Protein, CADD ( Computer Aided Drug Design)

\section{INTRODUCTION}

Dengue is fast emerging pandemic prone viral disease In many parts of the world.Dengue fever virus ( DENV) is an RNA virus of the family Flaviviridae and genus Flavivirus [Shasank Sekhar Swain, 2013]. The dengue virus is most significant arthropod born human pathogen, and increasing number of case have been reported over the last few decades.Dengue is caused via mosquito bite. They transfer the germs through saliva in the human body which binds to the human receptors thus causing dengue. The dengue virus genome contains about 11, 000 nucleotide bases [Muhamad awais amin et.al, 2015].

The NS5 protein from dengue virus is bifunctional and contains 900 amino acids. The S-adenosyl transferase activity resides within its $\mathrm{N}$-terminal domain and residue 270-900 from the RNA dependent RNA polymerase catalytic domain [Iranjan, Manoj, et.al, 2017]. Dengue virus contains four antigenically distinct viral serotypes named as DEN-1, DEN-2, DEN-3 and DEN-4 all the serotypes usually transmitted from one human host to the other by mosquitos of the Aedes genus, primarily Aedes egypti and Aedes albopictus, dengue virus possess_a unique serological investigations in mammals recommended that the circulation of dengue follows epidemiological patterns, without any noticeable clinical signs in host. while there is no licensed dengue drug and vaccine currently exists. Several candidates are currently undergoing either clinical evaluation or preclinical development. As a result there is need to design a dengue Vaccine, 
one of the antiviral agents approaches involves the blocking and inhibiting enzyme activity.[Sehrish Ruba, Mahira Arooj et.al, 2014].

The entry of dengue virus in the host cell is mediated by $\mathrm{E}$ protein, envelope protein present at the surface of virus enhances the fusion of viral cell membrane and host cell membrane. Initially a protein is arranged as heterodiamer in the immature state of dengue virus. The presence of premembrane protein which covers the protein peptide, will generate a spiky surface on E protein. The E protein monomer is composed of Barrales and organized into 3 structural domains domainI domainII, domain III. [Sajin.A.k.et.al, 2015]. The first reported case of dengue like illness in india was in madras in 1780 , the first viriologically proved epidemic of DF in india occurred in Calcutta and Eastern Coast of india in 1963-1964. DF is a severe flu like infection that involves individuals of all age groups ( infants, children, Adolescents, and adults). In this report we describe the molecular features of NS2B-NS3 protein in dengue infection in dengue by using bioinformatics approach like primary sequence analysis of protein by sequence retrieving from protein database. secondary structure prediction and understanding the basic secondary structure element study of tertiary structure by modelling 3D strcture using online tool like SWISS-MODEL. predicted 3D structure was validated by using PROCHECK analysis.

\section{METHODS AND MATERIAL}

\section{A) Retrieval of protein sequence information of NS2B-NS3}

For the study of NS2B-NS3 molecule its amino acid sequence retrieved from the major protein sequence database like UniprotKB and NCBI protein database. The uniprotknowledgebase ( UniprotKB) is the central hub for the collection of functional information on proteins, with accurate, consistent and rich annotation. The sequence obtained was stored in FASTA fromat with its accession number.

\section{B) Analysis of physicochemical parameters}

The analysis of physicochemical parameters of NS2BNS3 was done using online tool for protein prediction is protparam.It gives detailed information of protein like isoelectric point, theoretical PI, extinction coefficient, half life, instability index, aliphatic index, Grand average, hydropathecity.

\section{C) Secondary structure prediction of NS2B-NS3}

The secondary structure prediction of NS2B-NS3 is carried out using online protein prediction tool SOPMA. Self optimized prediction method ( SOPMA ). It gives the information of Alpha helix, beta sheets, extended strands, random coils.

\section{D) Prediction of tertiary structure of NS2B-NS3}

The tertiary structure of NS2B-NS3 was obtained by using SWISS MODEL tool by selecting the template with high homology and with optimized parameters. The obtained structure was stored in PDB format for visualization.

\section{E) Visualization of tertiary structure of NS2B_NS3}

The predicted structure of NS2B-NS3 is visualized by using structure visualization tool Rasmol. visualization was done using different formats to understand structural features of NS2B-NS3.

F) Model validation of tertiary structure of NS2B-NS3 3D structure of NS2B-NS3 was validated by using PROCHECK statistics and Ramchandran plot analysis.

\section{RESULTS AND DISCUSSION}

\subsection{Retrieval of protein sequence information of} NS2B-NS3 
For the study of NS2B-NS3 protein sequence was retrieved from major protein sequence database like uniprotKB. accession number of sequence is stored and sequence length is found to be 3396 amino acid. Uniprot Id : P29990

\subsection{Analysis of physicochochemical parameters}

TABLE 1. Showing physicochemical parameters

\begin{tabular}{|c|c|}
\hline Parameters & Values \\
\hline Aliphatic index & 86.32 \\
\hline GRAVY & -0.229 \\
\hline Instability index & 37.32 \\
\hline Molecular weight & 379546.53 \\
\hline Theoretical PI & 8.75 \\
\hline Extinction coefficient & 607270 \\
\hline $\begin{array}{l}\text { Total number of } \\
\text { negatively charged } \\
\text { residues ( Asp+ Glu ) }\end{array}$ & 373 \\
\hline $\begin{array}{l}\text { Total number of } \\
\text { positively charged } \\
\text { residues ( Arg+ Lys ) }\end{array}$ & 410 \\
\hline
\end{tabular}

The analysis of physicochemical properties of protein is carried out by using online protein prediction tool is protparam analysis. It gives therotical PI on the basis of nature and unstable on the basis of instability index. According to positive score indicates that higher hydrophobicity. on the basis of GRAVY score it denotes the hydrophilicity. calculated parameters are shown in below table 1

\subsection{Secondary structure information of NS2B-NS3}

Secondary structure prediction of NS2B-NS3 was carried out using SOPMA and all other secondary element like alpha helix, beta sheets, random coils, and extended strands were predicted as shown in table below

TABLE 2. Showing secondary structure element

\begin{tabular}{|l|l|l|l|}
\hline $\begin{array}{l}\text { Secondary } \\
\text { structure element }\end{array}$ & $\begin{array}{l}\text { Amino } \\
\text { acid }\end{array}$ & Percentage & Residue \\
\hline Alpha helix (Hh) & 1324 & $39.04 \%$ & 73 \\
\hline $\begin{array}{l}\text { Extended } \\
\text { strands(Ee) }\end{array}$ & 639 & $18.84 \%$ & 14 \\
\hline Beta turn(Tt) & 232 & $6.84 \%$ & 5 \\
\hline $\begin{array}{l}\text { Random coil } \\
(\mathrm{Cc})\end{array}$ & 1196 & $35.27 \%$ & 65 \\
\hline
\end{tabular}

\subsection{Tertiary structure prediction of NS2B-NS3}

Tertiary structure prediction was carried out using SWISS-MODEL online tool for building a 3D model of protein by selecting template with high homology and optimized characteristics. The obtained structure was stored in PDB format for visualization.The details of template selected for structure prediction was shown below Table

TABLE 3. Showing template details for NS2B-NS3

\begin{tabular}{|l|l|l|l|}
\hline Name & Title & Identity & Oligostate \\
\hline 2bmf.2A & $\begin{array}{l}\text { RNA } \\
\text { HELICASE }\end{array}$ & $98.44 \%$ & Monomer \\
\hline 3j27.1.A & $\begin{array}{l}\text { Envelope } \\
\text { protein E }\end{array}$ & $97.98 \%$ & $\begin{array}{l}\text { Hetero } \\
\text { Hexamer }\end{array}$ \\
\hline 5k5m.1.A & $\begin{array}{l}\text { RNA } \\
\text { dependent } \\
\text { RNA } \\
\text { polymerase }\end{array}$ & $98.43 \%$ & Monomer \\
\hline
\end{tabular}




\subsection{Visualization of tertiary structure of NS2B-NS3}

Tertiary structure of NS2B-NS3 is visualized using structure visualization tool Rasmol. Visualization was done using different formats to understand structural features of NS2B-NS3.The model were represented in figure 1.

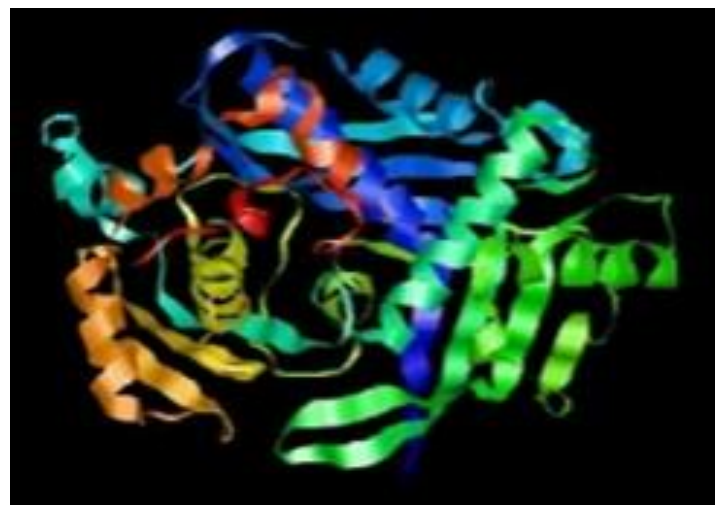

Figure1. showing 3D structure of NS2B-NS3

\subsection{Model validation using PROCHECK}

Using the procheck overall stereochemical quality of protein is evaluated by analysing the overall residue by residue geometry psi and phi torsion angles of Ramchandran plot analysis. It show residue with most favoured region, generously allowed region additionally allowed region and disallowed region. Ramchandran map of $2 \mathrm{FOM}$ showing in figure 2 below and table 4 shows procheck details given below.

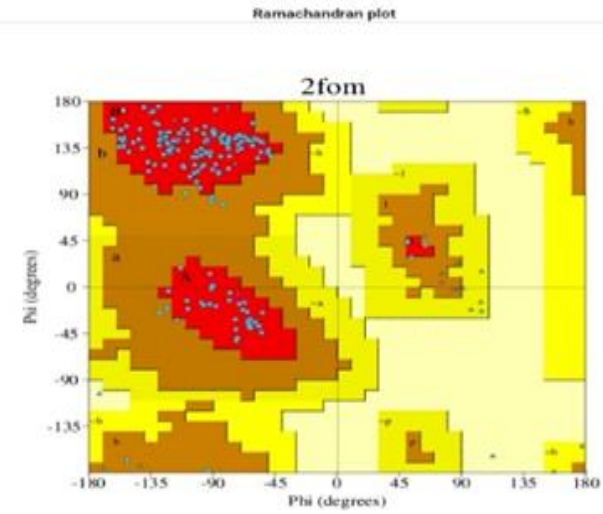

Figure 2. Showing Ramchandran plot analysis
TABLE 4. Showing Procheck Details

\begin{tabular}{|c|c|c|}
\hline $\begin{array}{l}\text { Procheck } \\
\text { statistics }\end{array}$ & No.of residues & Percentage $\%$ \\
\hline $\begin{array}{l}\text { Most favoured } \\
\text { regions }\end{array}$ & 344 & $89.04 \%$ \\
\hline $\begin{array}{l}\text { Generously } \\
\text { allowed } \\
\text { regions }\end{array}$ & 2 & $0.5 \%$ \\
\hline $\begin{array}{l}\text { Additional } \\
\text { allowed } \\
\text { regions }\end{array}$ & 39 & $10.1 \%$ \\
\hline $\begin{array}{l}\text { Non-glycine } \\
\text { and non- } \\
\text { proline residue }\end{array}$ & 385 & $100 \%$ \\
\hline $\begin{array}{l}\text { End-residue } \\
\text { (Gly and Pro) }\end{array}$ & 2 & \\
\hline $\begin{array}{l}\text { Glycine } \\
\text { residue }\end{array}$ & 27 & \\
\hline Proline residue & 441 & \\
\hline $\begin{array}{l}\text { Disallowed } \\
\text { regions }\end{array}$ & 0 & \\
\hline
\end{tabular}

\section{CONCLUSION}

Dengue fever is frequently born viral infection caused by female mosquito Aedes egypti. Transmission of dengue infection is transfer from one host to another by infected mosquito bite. Dengue virus is categorized into various serotypes on the basis of their genetic material variable. Dengue fever and dengue haemorrhagic fever is serious risk factor to Mankind. Nowdays there is no specific vaccine to treat the dengue infection. There is need to design potent antiviral vaccine against dengue fever. NS2B-NS3 act as potent drug target in dengue fever.

We conclude that NS2B-NS3 is potent drug target in dengue disease. This protein found in DENV 2 
infection.here we study all the molecular feature of protein like physicochemical properties, secondary structure prediction, tertiary structure prediction, and model validation using PROCHECK analysis.This all study gives preliminary information for designing a new drug with the help of computer based method like computer aided drug design ( CADD).

\section{REFERENCES}

[1]. Shasank Sekhar Swain, Debasmita dudey (2013) Anti dengue medicinal plants : A Mini review. Journal of Pharmacognosy and Phytochemistry volume 1 issue 2 ,

[2]. Muhamad awais amin, muhamad umer Ali, et.al ( 2015), Anti-dengue Virus Drug Candidate Target NS5 Protein .journal of Trends in biosciences 8 ( 19 ), ISSN 0974-8431, 5159-5166

[3]. Iranjan, Manoj Kumar, Suhasini Bhatnagar ( 2017) Identification of novel inhibitor against dengue NS5 .International journal of advances in Pharmaceutics 06(07):114-118 ISSN 2320-4931

[4]. Sehrish Ruba, Mahira Arooj et.al, (2014) in silico molecular docking studies and design of dengue virus Inhibitors IOSR journal of pharmacy and Biological Sciences Volume 9, issue, 2Ver pp 1523 ISSN 2319-7676

[5]. Sajin.A.k.et.al, (2015) Molecular docking studies on phytocompounds from the methanol leaf extracts of carica papaya against Envelope protein of dengue virus (type 2) Scholars research library, 5(2):1-7 ISSN 2231-3176

[6]. Shamimul Hasan, Sami Faisal Jamdar, Munther Alalowi, and Sadun Mohammad Al Ageel Al Beaiji ( 2016) Dengue virus: A global human threat: Review of literature journal of international society of preventive and community dentistry.

\section{Cite this article as :}

Vinod P. Sinoorkar, Snehal M. Mathe, Neha Guttikonda, "In Silico Analysis and Molecular Modelling of NS2B-NS3 Protein in Dengue", International Journal of Scientific Research in Science and Technology (IJSRST), Online ISSN : 2395-602X, Print ISSN : 2395-6011, Volume 6 Issue 2, pp. 526-530, March-April 2019. Available at doi : https://doi.org/10.32628/IJSRST1962101 Journal URL : http://ijsrst.com/IJSRST1962101 